CERN-PPE/93-91

04 June 1993

\title{
Search for Massive, Unstable Photinos that Violate R Parity
}

\author{
OPAL Collaboration
}

\begin{abstract}
A search for unstable, massive photinos pair produced in $e^{+} e^{-}$collisions has been performed using the OPAL detector at LEP. The data sample corresponds to an integrated luminosity of $21 \mathrm{pb}^{-1}$ collected at center-of-mass energies near the $Z^{0}$ resonance. The unstable photino is assumed to decay into $\tau^{ \pm} l^{\mp} \nu_{l}(l=\mathrm{e}$ or $\mu)$ final states, thereby violating individual lepton numbers and $\mathrm{R}$ parity. This search is inspired by a particular model of supersymmetry which is of interest within the context of solar neutrino experiments because it allows for a large neutrino magnetic moment. The present search excludes a large portion of the parameter space allowed by the model.
\end{abstract}

To be submitted to Physics Letters B 


\section{The OPAL Collaboration}

P.D. Acton ${ }^{25}$, R. Akers ${ }^{16}$, G. Alexander ${ }^{23}$, J. Allison ${ }^{16}$, K.J. Anderson ${ }^{9}$, S. Arcelli ${ }^{2}$, A. Astbury ${ }^{28}$, D. Axen ${ }^{29}$, G. Azuelos ${ }^{18, a}$, J.T.M. Baines ${ }^{16}$, A.H. Ball17, J. Banks ${ }^{16}$, R.J. Barlow ${ }^{16}$, S. Barnett ${ }^{16}$, R. Bartoldus ${ }^{3}$, J.R. Batley ${ }^{5}$, G. Beaudoin ${ }^{18}$, A. Beck ${ }^{23}$, G.A. Beck ${ }^{13}$, J. Becker ${ }^{10}$, C. Beeston ${ }^{16}$, T. Behnke ${ }^{27}$, K.W. Bell ${ }^{20}$, G. Bella ${ }^{23}$,

P. Bentkowski ${ }^{18}$, P. Berlich ${ }^{10}$, S. Bethke ${ }^{11}$, O. Biebel ${ }^{3}$, I.J. Bloodworth ${ }^{1}$, P. Bock ${ }^{11}$, B. Boden ${ }^{3}$, H.M. Bosch ${ }^{11}$, M. Boutemeur ${ }^{18}$, H. Breuker ${ }^{8}$, P. Bright-Thomas ${ }^{25}$, R.M. Brown ${ }^{20}$, A. Buijs ${ }^{8}$, H.J. Burckhart ${ }^{8}$, C. Burgard ${ }^{27}$, P. Capiluppi ${ }^{2}$, R.K. Carnegie ${ }^{6}$, A.A. Carter ${ }^{13}$, J.R. Carter ${ }^{5}$, C.Y.Chang ${ }^{17}$, D.G. Charlton ${ }^{8}$, S.L. Chu ${ }^{4}$, P.E.L. Clarke ${ }^{25}$, J.C. Clayton ${ }^{1}$, I. Cohen ${ }^{23}$, J.E. Conboy ${ }^{15}$, M. Cooper ${ }^{22}$, M. Coupland ${ }^{14}$, M. Cuffiani ${ }^{2}$, S. Dado ${ }^{22}$, G.M. Dallavalle ${ }^{2}$, S. De Jong ${ }^{13}$, L.A. del Pozo ${ }^{5}$, H. Deng ${ }^{17}$, A. Dieckmann ${ }^{11}$,

M. Dittmar ${ }^{4}$, M.S. Dixit ${ }^{7}$, E. do Couto e Silva ${ }^{12}$, J.E. Duboscq ${ }^{8}$, E. Duchovni ${ }^{26}$, G. Duckeck ${ }^{11}$, I.P. Duerdoth ${ }^{16}$, D.J.P. Dumas ${ }^{6}$, P.A. Elcombe ${ }^{5}$, P.G. Estabrooks ${ }^{6}$, E. Etzion ${ }^{23}$, H.G. Evans ${ }^{9}$, F. Fabbri ${ }^{2}$, B. Fabbro ${ }^{21}$, M. Fierro ${ }^{2}$, M. Fincke-Keeler ${ }^{28}$, H.M. Fischer ${ }^{3}$, D.G. Fong ${ }^{17}$, M. Foucher ${ }^{17}$, A. Gaidot ${ }^{21}$, J.W. Gary ${ }^{4}$, J. Gascon ${ }^{18}$,

N.I. Geddes ${ }^{20}$, C. Geich-Gimbel ${ }^{3}$, S.W. Gensler ${ }^{9}$, F.X. Gentit ${ }^{21}$, G. Giacomelli ${ }^{2}$, R. Giacomelli ${ }^{2}$, V. Gibson ${ }^{5}$, W.R. Gibson ${ }^{13}$, J.D. Gillies ${ }^{20}$, J. Goldberg ${ }^{22}$,

D.M. Gingrich ${ }^{30, a}$, M.J. Goodrick ${ }^{5}$, W. Gorn ${ }^{4}$, C. Grandi ${ }^{2}$, F.C. Grant ${ }^{5}$, J. Hagemann ${ }^{27}$, G.G. Hanson ${ }^{12}$, M. Hansroul ${ }^{8}$, C.K. Hargrove ${ }^{7}$, P.F. Harrison ${ }^{13}$, J. Hart ${ }^{8}$,

P.M. Hattersley ${ }^{1}$, M. Hauschild ${ }^{8}$, C.M. Hawkes ${ }^{8}$, E. Heflin ${ }^{4}$, R.J. Hemingway ${ }^{6}$, G. Herten ${ }^{10}$, R.D. Heuer ${ }^{8}$, J.C. Hill ${ }^{5}$, S.J. Hillier ${ }^{8}$, T. Hilse ${ }^{10}$, D.A. Hinshaw ${ }^{18}$, J.D. Hobbs ${ }^{8}$, P.R. Hobson ${ }^{25}$, D. Hochman ${ }^{26}$, R.J. Homer ${ }^{1}$, A.K.Honma ${ }^{28, a}$,

R.E. Hughes-Jones ${ }^{8}$, R. Humbert ${ }^{10}$, P. Igo-Kemenes ${ }^{11}$, H. Ihssen ${ }^{11}$, D.C. Imrie ${ }^{25}$, A.C. Janissen ${ }^{6}$, A. Jawahery ${ }^{17}$, P.W. Jeffreys ${ }^{20}$, H. Jeremie ${ }^{18}$, M. Jimack ${ }^{1}$, M. Jones ${ }^{29}$,

R.W.L. Jones ${ }^{8}$, P. Jovanovic ${ }^{1}$, C. Jui ${ }^{4}$, D. Karlen ${ }^{6}$, K. Kawagoe ${ }^{24}$, T. Kawamoto ${ }^{24}$, R.K. Keeler ${ }^{28}$, R.G. Kellogg ${ }^{17}$, B.W. Kennedy ${ }^{15}$, S. Kluth ${ }^{5}$, T. Kobayashi ${ }^{24}$, D.S. Koetke ${ }^{8}$, T.P. Kokott ${ }^{3}$, S. Komamiya ${ }^{24}$, L. Köpke ${ }^{8}$, J.F. Kral ${ }^{8}$, R. Kowalewski ${ }^{6}$, J. von Krogh ${ }^{11}$, J. Kroll ${ }^{9}$, M. Kuwano ${ }^{24}$, P. Kyberd ${ }^{13}$, G.D. Lafferty ${ }^{16}$, H. Lafoux ${ }^{21}$, R. Lahmann ${ }^{17}$, F. Lamarche ${ }^{18}$, J.G. Layter ${ }^{4}$, P. Leblanc ${ }^{18}$, A.M. Lee ${ }^{31}$, M.H. Lehto ${ }^{15}$, D. Lellouch ${ }^{26}$, C. Leroy ${ }^{18}$, J. Letts ${ }^{4}$, S. Levegrün ${ }^{3}$, L. Levinson ${ }^{26}$, S.L. Lloyd ${ }^{13}$, F.K. Loebinger ${ }^{16}$, J.M. Lorah ${ }^{17}$, B. Lorazo ${ }^{18}$, M.J. Losty ${ }^{7}$, X.C. Lou ${ }^{12}$, J. Ludwig ${ }^{10}$, A. Luig ${ }^{10}$, M. Mannelli ${ }^{8}$, S. Marcellini' ${ }^{2}$, C. Markus ${ }^{3}$, A.J. Martin ${ }^{13}$, J.P. Martin ${ }^{18}$, T. Mashimo ${ }^{24}$, P. Mättig ${ }^{3}$, U. Maur ${ }^{3}$, J. McKenna ${ }^{28}$, T.J. McMahon ${ }^{1}$, J.R. McNutt ${ }^{25}$, F. Meijers ${ }^{8}$, D. Menszner ${ }^{11}$, F.S. Merritt ${ }^{9}$, H. Mes $^{7}$, A. Michelini ${ }^{8}$, R.P. Middleton ${ }^{20}$, G. Mikenberg ${ }^{26}$,

J. Mildenberger ${ }^{6}$, D.J. Miller ${ }^{15}$, R. Mir ${ }^{12}$, W. Mohr ${ }^{10}$, C. Moisan ${ }^{18}$, A. Montanari ${ }^{2}$, T. Mori ${ }^{24}$, M. Morii ${ }^{24}$, U. Müller ${ }^{3}$, B. Nellen ${ }^{3}$, H.H. Nguyen ${ }^{9}$, S.W. O'Neale ${ }^{1}$,

F.G. Oakham ${ }^{7}$, F.Odorici ${ }^{2}$, H.O. Ogren ${ }^{12}$, C.J. Oram ${ }^{28, a}$, M.J.Oreglia ${ }^{9}$, S. Orito ${ }^{24}$, J.P. Pansart ${ }^{21}$, B. Panzer-Steindel ${ }^{8}$, P. Paschievici ${ }^{26}$, G.N.Patrick ${ }^{20}$, N. Paz-Jaoshvili ${ }^{23}$, M.J.Pearce ${ }^{1}$, P. Pfister ${ }^{10}$, J.E. Pilcher ${ }^{9}$, J. Pinfold ${ }^{30}$, D. Pitman ${ }^{28}$, D.E. Plane ${ }^{8}$, P. Poffenberger ${ }^{28}$, B. Poli ${ }^{2}$, A. Pouladdej ${ }^{6}$, T.W. Pritchard ${ }^{13}$, H. Przysiezniak ${ }^{18}$, 
G. Quast ${ }^{27}$, M.W. Redmond ${ }^{8}$, D.L. Rees $^{8}$, G.E. Richards ${ }^{16}$, S.A. Robins ${ }^{13}$, D. Robinson ${ }^{8}$, A. Rollnik ${ }^{3}$, J.M. Roney ${ }^{28, b},{\text { E. } \operatorname{Ros}^{8}, \text { S. Rossberg }}^{10}$, A.M. Rossi ${ }^{2}$, M. Rosvick ${ }^{28}$, P. Routenburg ${ }^{6}$, K. Runge ${ }^{10}$, O. Runolfsson ${ }^{8}$, D.R. Rust ${ }^{12}$, M. Sasaki ${ }^{24}$, C.Sbarra ${ }^{2}$, A.D. Schaile ${ }^{10}$, O.Schaile ${ }^{10}$, W.Schappert ${ }^{6}$, P.Scharff-Hansen ${ }^{8}$, P. Schenk ${ }^{4}$, B. Schmitt ${ }^{3}$, H. von der Schmitt ${ }^{11}$, M. Schröder ${ }^{12}$, C.Schwick ${ }^{27}$, J.Schwiening ${ }^{3}$, W.G. Scott ${ }^{20}$,

M.Settles ${ }^{12}$, T.G. Shears ${ }^{5}$, B.C.Shen ${ }^{4}$, C.H.Shepherd-Themistocleous ${ }^{7}$, P. Sherwood ${ }^{15}$, G.P.Siroli ${ }^{2}$, A.Skillman ${ }^{16}$, A.Skuja ${ }^{17}$, A.M.Smith ${ }^{8}$, T.J.Smith ${ }^{28}$, G.A.Snow ${ }^{17}$, R.Sobie ${ }^{28, b}$, R.W.Springer ${ }^{17}$, M.Sproston ${ }^{20}$, A.Stahl ${ }^{3}$, C.Stegmann ${ }^{10}$, K.Stephens ${ }^{16}$, J.Steuerer ${ }^{28}$, R.Ströhmer ${ }^{11}$, D. Strom ${ }^{19}$, H. Takeda ${ }^{24}$, T. Takeshita ${ }^{24, c}$, S. Tarem ${ }^{26}$, M. Tecchio ${ }^{9}$, P. Teixeira-Dias ${ }^{11}$, N. Tesch ${ }^{3}$, M.A.Thomson ${ }^{15}$, E. Torrente-Lujan ${ }^{22}$, S. Towers ${ }^{28}$, G. Transtromer ${ }^{25}$, N.J. Tresilian ${ }^{16}$, T. Tsukamoto ${ }^{24}$, M.F. Turner ${ }^{8}$, D. Van den plas ${ }^{18}$, R. Van Kooten ${ }^{27}$, G.J. VanDalen ${ }^{4}$, G. Vasseur ${ }^{21}$, C.J. Virtue ${ }^{7}$, A. Wagner ${ }^{27}$, D.L. Wagner ${ }^{9}$, C. Wahl ${ }^{10}$, C.P. Ward ${ }^{5}$, D.R. Ward ${ }^{5}$, P.M. Watkins ${ }^{1}$, A.T. Watson ${ }^{1}$, N.K. Watson ${ }^{8}$, M. Weber ${ }^{11}$, P. Weber ${ }^{6}$, P.S. Wells ${ }^{8}$, N. Wermes ${ }^{3}$, M.A. Whalley ${ }^{1}$, B. Wilkens ${ }^{10}$, G.W.Wilson ${ }^{4}$, J.A. Wilson ${ }^{1}$, V-H. Winterer ${ }^{10}$, T. Wlodek ${ }^{26}$, G. Wolf ${ }^{26}$, S. Wotton ${ }^{11}$, T.R. Wyatt ${ }^{16}$, R. Yaari ${ }^{26}$, A. Yeaman ${ }^{13}$, G. Yekutieli ${ }^{26}$, M. Yurko ${ }^{18}$, W. Zeuner ${ }^{8}$, G.T. Zorn ${ }^{17}$.

${ }^{1}$ School of Physics and Space Research, University of Birmingham, Birmingham, B15 2TT, UK

${ }^{2}$ Dipartimento di Fisica dell' Università di Bologna and INFN, Bologna, 40126, Italy

${ }^{3}$ Physikalisches Institut, Universität Bonn, D-5300 Bonn 1, Germany

${ }^{4}$ Department of Physics, University of California, Riverside, CA 92521 USA

${ }^{5}$ Cavendish Laboratory, Cambridge, CB3 0HE, UK

${ }^{6}$ Carleton University, Dept of Physics, Colonel By Drive, Ottawa, Ontario K1S 5B6, Canada

${ }^{7}$ Centre for Research in Particle Physics, Carleton University, Ottawa, Ontario K1S 5B6, Canada

${ }^{8}$ CERN, European Organisation for Particle Physics, 1211 Geneva 23, Switzerland

${ }^{9}$ Enrico Fermi Institute and Dept of Physics, University of Chicago, Chicago Illinois 60637, USA

${ }^{10}$ Fakultät für Physik, Albert Ludwigs Universität, D-7800 Freiburg, Germany

${ }^{11}$ Physikalisches Institut, Universität Heidelberg, Heidelberg, Germany

${ }^{12}$ Indiana University, Dept of Physics, Swain Hall West 117, Bloomington, Indiana 47405, USA

${ }^{13}$ Queen Mary and Westfield College, University of London, London, E1 4NS, UK

${ }^{14}$ Birkbeck College, London, WC1E 7HV, UK

${ }^{15}$ University College London, London, WC1E 6BT, UK

${ }^{16}$ Department of Physics, Schuster Laboratory, The University, Manchester, M13 9PL, UK

${ }^{17}$ Department of Physics, University of Maryland, College Park, Maryland 20742, USA 
${ }^{18}$ Laboratoire de Physique Nucléaire, Université de Montréal, Montréal, Quebec, H3C 3J7, Canada

${ }^{19}$ University of Oregon, Dept of Physics, Eugene, Oregon 97403, USA

${ }^{20}$ Rutherford Appleton Laboratory, Chilton, Didcot, Oxfordshire, OX11 0QX, UK

${ }^{21}$ DAPNIA/SPP, Saclay, F-91191 Gif-sur-Yvette, France

${ }^{22}$ Department of Physics, Technion-Israel Institute of Technology, Haifa 32000, Israel

${ }^{23}$ Department of Physics and Astronomy, Tel Aviv University, Tel Aviv 69978, Israel

${ }^{24}$ International Centre for Elementary Particle Physics and Dept of Physics, University of Tokyo, Tokyo 113, and Kobe University, Kobe 657, Japan

${ }^{25}$ Brunel University, Uxbridge, Middlesex, UB8 3PH UK

${ }^{26}$ Nuclear Physics Department, Weizmann Institute of Science, Rehovot, 76100, Israel

${ }^{27}$ Universität Hamburg/DESY, II Inst für Experimental Physik, 2000 Hamburg 52, Germany

${ }^{28}$ University of Victoria, Dept of Physics, P O Box 3055, Victoria BC V8W 3P6, Canada

${ }^{29}$ University of British Columbia, Dept of Physics, Vancouver BC V6T 1Z1, Canada

${ }^{30}$ University of Alberta, Dept of Physics, Edmonton AB T6G 2N5, Canada

${ }^{31}$ Duke University, Dept of Physics, Durham, North Carolina 27708-0305, USA

${ }^{a}$ Also at TRIUMF, Vancouver, Canada V6T $2 \mathrm{~A} 3$

${ }^{b}$ And IPP, University of Victoria, Dept of Physics, P O Box 3055, Victoria BC V8W 3P6, Canada

${ }^{c}$ Also at Shinshu University, Matsumoto 390, Japan 


\section{Introduction}

The apparent anticorrelation of the solar-neutrino signal with the 11 year sunspot cycle reported by Davis[1] may be explained if the electron neutrino has a large magnetic moment $[2]\left(\approx 10^{-11} \mu_{B}\right.$, where $\mu_{B}$ is the proton Bohr magneton $)$. Babu and Mohapatra[3] have shown how this might be achieved within the framework of an unusual but relatively simple supersymmetric extension of the Standard Model with $\mathrm{R}$ parity violating interactions. In this model the neutrinos have a large magnetic moment, and therefore a $\nu_{e}$ can transform into a $\nu_{\mu}$ in the sun's magnetic field, thus reducing the number of $\nu_{e}$ expected to reach the earth. According to the model, the individual lepton numbers, $L_{e}$, $L_{\mu}$ and $L_{\tau}$, are not conserved while the difference $L_{e}-L_{\mu}$ remains a conserved quantum number. The $L_{e}-L_{\mu}$ symmetry forbids the occurrence of unobserved processes such as $\mu \rightarrow e \gamma$ and $\mu \rightarrow 3 e$ as well as neutrinoless double beta decay. Other rare processes such as $\mu^{-}+A \rightarrow e^{+}+B$ are allowed by $L_{e}-L_{\mu}$ symmetry but would occur only in higher orders because the model does not explicitly introduce doubly charged particles. In the Babu-Mohapatra model, unlike in many supersymmetric extensions of the Standard Model, the large magnetic moment of the neutrino restricts the selectron mass, $M_{\tilde{e}}$, to less than about $100 \mathrm{GeV} / \mathrm{c}^{2}$. Direct searches[4] for $\tilde{e}$ at LEP and SLC have excluded the range $M_{\tilde{e}}<43 G e V / c^{2}$, but only by assuming a stable, invisible photino. Hence these limits are not valid for the model considered here.

We present a search for the reaction

$$
e^{+} e^{-} \rightarrow \tilde{\gamma} \tilde{\gamma}
$$

where $\tilde{\gamma}$ is the lightest supersymmetric particle, assumed by the model to be a pure photino. Consequences arising from a possible mixing with zino or higgsino states will be discussed later. Assuming a pure photino, the decay $Z^{0} \rightarrow \tilde{\gamma} \tilde{\gamma}$ is forbidden. The reaction proceeds by the exchange of a selectron (see figure 1a), and the cross section depends on the selectron mass, $M_{\tilde{e}}$. Following[3] we assume that the photino decays via the following channels, which violate $\mathrm{R}$ parity and individual lepton number conservation but conserve $\left(L_{e}-L_{\mu}\right)$ :

$$
\begin{aligned}
\tilde{\gamma} & \rightarrow \tau^{+} e^{-} \nu_{\mu}, \\
& \rightarrow \tau^{-} e^{+} \bar{\nu}_{\mu}, \\
& \rightarrow \tau^{+} \mu^{-} \nu_{e}, \\
& \rightarrow \tau^{-} \mu^{+} \bar{\nu}_{e} .
\end{aligned}
$$

The present search is limited by the available center-of-mass energy to $2 M_{\tilde{\gamma}} \leq M_{Z^{0}}$. 
Searches for the analogous channel $e^{+} e^{-} \rightarrow \tilde{\gamma} \tilde{\gamma} \gamma$ have been carried out at PETRA, PEP and TRISTAN for stable photinos. For example, one of the best limits was obtained by the ASP experiment[5], which finds that $M_{\tilde{e}} \geq 40 \mathrm{GeV} / \mathrm{c}^{2}$ for $M_{\tilde{\gamma}} \leq 10 \mathrm{GeV} / \mathrm{c}^{2}(90 \%$ CL). However, since the photino was assumed to be stable, these limits do not apply for the model considered here.

We describe a search for unstable photinos in the mass range $5 \mathrm{GeV} / \mathrm{c}^{2}<M_{\tilde{\gamma}}<$ $45 \mathrm{GeV} / c^{2}$ and $M_{\tilde{e}} \geq M_{\tilde{\gamma}}$ via the production and decay processes (1) and (2), the diagrams of which are shown in figure 1 . For $M_{\tilde{\gamma}}<5 \mathrm{GeV} / \mathrm{c}^{2}$, the photino pairs cannot be separated from $\tau$ pairs and other backgrounds. The $e^{+} e^{-} \rightarrow \tilde{\gamma} \tilde{\gamma}$ events would have a simple topology and distinctive signatures: a low charged particle multiplicity and at least one energetic charged lepton in each event hemisphere (the two hemispheres are defined by the plane perpendicular to the thrust axis containing the $e^{+} e^{-}$interaction point). The events would also have substantial missing energy. Following reference[3] we assume that the photino has a lifetime $<10^{-12}$ seconds and thus decays close to the $e^{+} e^{-}$interaction point. Since the decays proceed through the exchange of massive scalar particles, the angular and energy distributions of the primary leptons from $\tilde{\gamma}$ decays would differ from those of secondary leptons from $\tau$ decays.

\section{The OPAL Detector}

The OPAL detector[6] has a geometrical acceptance close to $4 \pi$ steradians. The central part consists of a set of tracking detectors in a $0.435 \mathrm{~T}$ solenoidal magnetic field. It is surrounded successively by a time-of-flight scintillation counter array, a lead glass electromagnetic calorimeter with presampler, an instrumented iron magnet yoke which serves as a hadron calorimeter, and finally by a set of muon chambers. Two identical forward detectors, serving to monitor the luminosity, are mounted close to the beam axis at either side of the interaction point.

The central tracking detector consists of a precision vertex chamber surrounding the beam-pipe, and a large volume jet chamber, for precise tracking in the $r$ - $\phi$ plane. This is followed by a set of $z$-chambers for tracking in the $r$ - $z$ plane (the coordinate system is defined with $+z$ along the $e^{-}$beam, $\theta$ and $\phi$ being the polar and azimuthal angles, respectively). Besides tracking and momentum measurements, the central detector also contributes to particle identification by the measurement of the specific ionization loss $(\mathrm{d} E / \mathrm{d} x)$ of charged particles.

The electromagnetic calorimeter consists of a cylindrical array (barrel part) and two endcap arrays of 12,000 lead glass blocks in total. Each block is approximately 25 radiation lengths thick, and covers a solid angle of approximately 40 x $40 \mathrm{mrad}^{2}$. The hadron calorimeter consists of nine (eight) layers of iron plates in the barrel (endcap) region, each $10 \mathrm{~cm}$ thick, giving a total thickness of about 4.5 interaction lengths. The iron plates alternate with layers of limited streamer tubes having both pad and strip read- 
out. Besides measuring hadronic energy, the hadron calorimeter also serves to identify muons using the strip readout information of successive layers. The muon detector which surrounds the hadron calorimeter consists of four layers of drift chambers in the barrel region and four layers of limited streamer tubes in the endcap region. The luminosity of the colliding beams is obtained from detecting small angle Bhabha scattering in the two forward detectors which consist of lead-scintillator calorimeters with associated tracking chambers and subtend the angular range $40<\theta<150 \mathrm{mrad}$.

\section{Event simulation}

Events were generated to simulate the reaction $e^{+} e^{-} \rightarrow \tilde{\gamma} \tilde{\gamma}$. The generator used analytic formulae for the production and decay reactions, as provided by the authors of the model[3]. We have assumed equal mass for all charged scalar leptons $\left(M_{\tilde{e}}=M_{\tilde{\mu}}=\right.$ $M_{\tilde{\tau}}$ ). However, we have also verified that relaxing this assumption by allowing $M_{\tilde{e}}=M_{\tilde{\mu}}$ to differ from $M_{\tilde{\tau}}$ by a factor of two in either direction does not significantly alter the results. This effect is included in the systematic error. The generated events were put through the simulation programs describing the response of the OPAL detector[7]. One million events were processed with a fast detector simulation, and 9000 events with a detailed detector simulation.

The various background processes were simulated using JETSET 7.3[8] for multihadronic $Z^{0}$ decays and KORALZ 3.8 [9] for tau and muon pairs. The program described in reference[10] was used to generate Bhabha events. Four-fermion final states from $e^{+} e^{-} \rightarrow \ell^{+} \ell^{-} \ell^{\prime+} \ell^{\prime-}$ (with $\ell, \ell^{\prime}=e, \mu, \tau$ ) were simulated using the generator described in reference[11] in a manner discussed in detail in reference[12]. The number of generated events for each mode was at least as large as that expected in the data sample.

\section{Event Selection}

The data used in this analysis were accumulated during 1990 and 1991 and correspond to an integrated luminosity of $21.0 \mathrm{pb}^{-1}$ or about 500,000 hadronic $Z^{0}$ decays. Charged tracks were labeled as "good" if they had at least 40 hits in the jet chamber (which has 159 layers of sense wires), transverse momentum $p_{\perp}>200 \mathrm{MeV} / \mathrm{c}$, polar angle | $\cos \theta \mid<0.94$, transverse distance from the interaction point $<2 \mathrm{~cm}$, and longitudinal distance $<40 \mathrm{~cm}$. Electrons were identified by requiring that the charged track has a matching electromagnetic cluster and that the cluster energy, $E$, and track momentum, $p$, satisfy $0.75<E / p<1.5$. Muons were identified as central detector tracks with matching hits in the muon chamber or with matching track segments in the hadron calorimeter using the strip readout as described in reference[13]. Any pair of tracks with opposite charge outside a $30^{\circ}$ cone around the thrust axis and having an invariant mass less than $0.2 \mathrm{GeV} / \mathrm{c}^{2}$ was considered as a photon conversion into an $e^{+} e^{-}$pair, and was removed from the track count.

The selection criteria are described below and their effect on the data, the simulated 
background, and a simulated signal sample (with mass assignments $M_{\tilde{e}}=70 \mathrm{GeV} / \mathrm{c}^{2}$ and $M_{\tilde{\gamma}}=20 \mathrm{GeV} / \mathrm{c}^{2}$ ) are listed in Table 1 .

To eliminate badly measured events we required that the number of "good" tracks exceed $50 \%$ of all tracks (Selection A). Among the "good" tracks at least two had to be identified as charged leptons (e or $\mu$ ) with momentum $>3 G e V / c$ and satisfying $|\cos \theta| \leq 0.90$ (Selection B). Each event hemisphere had to contain at least one such lepton. In order to select events with the signal topology we required at least four and not more than eight "good" tracks (Selection C).

As can be seen in Table I, the above requirements eliminated more than $98 \%$ of the $Z^{0}$ events, while keeping a high selection efficiency for the signal. For example, taking $M_{\tilde{e}}=70 \mathrm{GeV} / \mathrm{c}^{2}$ and $M_{\tilde{\gamma}}=20 \mathrm{GeV} / \mathrm{c}^{2}$, the selection efficiency is $50.7 \%$.

The main backgrounds remaining are from $\tau^{+} \tau^{-}$and radiative $\mathrm{e}^{+} \mathrm{e}^{-}$and $\mu^{+} \mu^{-}$events. To eliminate most of these backgrounds we require that the event thrust be less than 0.98 (Selection D). The thrust distribution is shown in figure 2 for the data, the simulated background and for a simulated signal with $M_{\tilde{e}}=70 \mathrm{GeV} / \mathrm{c}^{2}$ and $M_{\tilde{\gamma}}=20 \mathrm{GeV} / \mathrm{c}^{2}$. We further required exactly two or exactly four tracks in at least one hemisphere (Selection E). The tau pair background was further reduced by requiring that the invariant mass in each hemisphere be greater than $2 \mathrm{GeV} / \mathrm{c}^{2}$. The invariant mass was calculated using charged tracks and high-energy ( $>1.5 \mathrm{GeV}$ for electromagnetic and $>10 \mathrm{GeV}$ for hadron) clusters not associated with the tracks. Since the signal events would contain a $\tau$ decay plus an energetic lepton in each hemisphere, we also required that the invariant mass in each hemisphere, without the lepton, be less than $2 \mathrm{GeV} / \mathrm{c}^{2}$. For events with more than one energetic lepton in the same hemisphere, each lepton was separately excluded, and the smallest invariant mass was used (Selection F).

Three events remain at this point, while the expected background is 3.1 events. Roughly half of the background arose from the four-fermion final state and was suppressed by requiring significant missing energy: $\Sigma|c \vec{p}| / \sqrt{s}<0.80$, where the sum runs over all "good" tracks in the event (Selection G). In figure 3 the distribution of this quantity is shown just before Selection G was applied. The three events in the data sample are indicated by stars. The four-lepton background simulation (dark hatched histogram) predicted 1.5 events of which only 0.85 events were expected to survive Selection G. The $Z^{0}$ multihadronic decays are expected to contribute 1.1 events (light hatched histogram). Figure 3 also shows the distribution of the 18 simulated signal events (unhatched histogram), after proper normalization, for $M_{\tilde{\gamma}}=20 \mathrm{GeV} / \mathrm{c}^{2}$ and $M_{\tilde{e}}=70 \mathrm{GeV} / \mathrm{c}^{2}$. No events remain in the data following Selection G.

For $M_{\tilde{\gamma}}$ below $10 \mathrm{GeV} / \mathrm{c}^{2}$, the detection efficiency falls rapidly due primarily to Selection D. To improve the sensitivity at low $M_{\tilde{\gamma}}$ we removed the thrust cut (Selection D), and modified Selection G, which was a cut on charged track energy alone, to become a 
cut on charged plus neutral energy; namely we require that the ratio of the total visible energy to the total available energy be less than 0.8 . One data event survives, while the expected background remained unchanged. Although the one surviving event is consistent with a $B_{0} \bar{B}_{0} X$ assignment (with both $\mathrm{B}$ mesons decaying leptonically) we consider it as a possible signal event when deriving the mass limits with the modified cuts.

\section{Results}

The negative outcome of this search is transformed into a lower limit for $M_{\tilde{e}}$ as a function of $M_{\tilde{\gamma}}$ in the framework of the Babu-Mohapatra model[3]. The selection efficiencies for the $\tilde{\gamma} \tilde{\gamma}$ signal in given points of the $\left(M_{\tilde{e}}, M_{\tilde{\gamma}}\right)$ plane are derived from a grid of 18 points using 50,000 fast Monte Carlo events per point. The fast simulation was compared at nine grid points with 1000 full Monte Carlo events per point. The change of efficiency was included in the systematic error. For example, for $M_{\tilde{e}}=70 \mathrm{GeV} / \mathrm{c}^{2}$ and $M_{\tilde{\gamma}}=20 \mathrm{GeV} / \mathrm{c}^{2}$ the final selection efficiency was $32.5 \pm 1.0 \%$ (the error is statistical).

Systematic uncertainties arise from: the determination of the integrated luminosity $(2 \%)$; initial and final state radiation (2\%); electron and muon detection efficiencies $(4 \%)$ (For more details see Ref.[14]). Variation of the selection criteria introduced an additional uncertainty of $2.5 \%$. The theoretical uncertainty due to varying the ratio of $M_{\tilde{\epsilon}, \tilde{\mu}}, / M_{\tilde{\tau}}$ contributes $1 \%$. Combining these uncertainties in quadrature gives a total systematic error of $5.6 \%$. To derive mass limits in the $\left(M_{\tilde{e}}, M_{\tilde{\gamma}}\right)$ plane we reduced the detection efficiencies by their total error which amounts to $6.3 \%$.

Table 2 shows the lower limit obtained in this way for the number of expected events for various photino and selectron masses. Since in the search above $M_{\tilde{\gamma}}=10 \mathrm{GeV} / \mathrm{c}^{2}$ no event was observed, the $95 \%$ CL limit was put to those points of the $\left(M_{\tilde{\epsilon}}, M_{\tilde{\gamma}}\right)$ plane where the number of expected events was 3.0 while in the search below $M_{\tilde{\gamma}}=10 \mathrm{GeV} / \mathrm{c}^{2}$ where one possible signal event was observed the limit was set at 4.7 events.

Figure 4 displays the $95 \%$ CL exclusion curve as a function of $M_{\tilde{\gamma}}$ and $M_{\tilde{e}}$. The present search excludes a large domain in the $\left(M_{\tilde{e}}, M_{\tilde{\gamma}}\right)$ plane allowed by the model of Babu and Mohapatra. Note that a $10 \%$ variation in the cross section prediction would move the limit on the selectron mass (for fixed photino mass) by less than $4 \%$. The present exclusion does not take into account additional theoretical uncertainties which may arise from the possibility that the lightest neutralino, $\chi^{0}$, is not a pure photino. If the $\chi^{0}$ has an important zino or higgsino component, our conclusion can be weakened, unless the dominant $\chi^{0}$ decays remain those listed in (2). In the latter case, a new class of events could be produced at the $Z^{0}$ resonance, namely $Z^{0} \rightarrow \chi^{0} \chi^{0}$ and $\chi^{0} \chi^{\prime 0}$, followed by $\chi^{0}$ decaying through its $\tilde{\gamma}$ component. Such events would constitute an important background to the Babu-Mohapatra process. However, since we do not observe such events, our limits are not affected by this scenario. 


\section{Summary}

A search has been carried out for unstable photinos that decay into leptons according to a specific model of supersymmetry conjectured by Babu and Mohapatra. In this model neutrinos have a large transition magnetic moment and could thereby explain the possible correlation of solar neutrino events with sunspot activity. No evidence for such photinos was found. A large region of the $\left(M_{\tilde{\epsilon}}, M_{\tilde{\gamma}}\right)$ plane which would be allowed by the Babu-Mohapatra model is excluded by the present search.

\section{Acknowledgements}

It is a pleasure to thank the SL Division for their efficient operation of the LEP machine and their continuing close cooperation with our experimental group. Numerous discussions with Professor Mohapatra and Dr. Babu are gratefully acknowledged. In addition to the support staff at our institutions we are pleased to acknowledge the following:

Department of Energy, USA,

National Science Foundation, USA,

Texas National Research Laboratory Commission, USA,

Science and Engineering Research Council, UK,

Natural Sciences and Engineering Research Council, Canada,

Fussefeld Foundation,

Israeli Ministry of Energy and Ministry of Science,

Minerva Gesellschaft,

Japanese Ministry of Education, Science and Culture (the Monbusho) and a grant under the Monbusho International Science Research Program,

German Israeli Bi-national Science Foundation (GIF),

Direction des Sciences de la Matière du Commissariat à l'Energie Atomique, France,

Bundesministerium für Forschung und Technologie, Germany,

National Research Council of Canada,

A.P. Sloan Foundation and Junta Nacional de Investigação Científica e Tecnológica, Portugal. 
Table 1. Number of events that survive successive selection criteria, A to G, described in the text. The Monte Carlo background simulation includes $Z^{0}$ decays into hadrons, lepton pairs and four lepton final states. The signal efficiency shown here corresponds to $M_{\tilde{e}}=70 \mathrm{GeV} / \mathrm{c}^{2}$ and $M_{\tilde{\gamma}}=20 \mathrm{GeV} / \mathrm{c}^{2}$.

\begin{tabular}{|c|c|c|c|}
\hline \multirow{2}{*}{$\begin{array}{l}\text { Selection } \\
\text { Criteria }\end{array}$} & \multicolumn{2}{|c|}{$\begin{array}{l}\text { Number of events that survive } \\
\text { the successive selection criteria }\end{array}$} & \multirow{2}{*}{$\begin{array}{l}\text { Signal } \\
\text { Efficiency }\end{array}$} \\
\hline & $\begin{array}{r}\text { OPAL } \\
\text { Data }\end{array}$ & $\begin{array}{l}\text { Monte Carlo } \\
\text { Background }\end{array}$ & \\
\hline (A) Preselected Sample & $305 \quad 021$ & 98981 & 81.9 \\
\hline (B) Energetic e or $\mu$ & 28444 & 10732 & 60.8 \\
\hline (C) 4 to 8 "good" tracks & 1163 & 1431 & 50.7 \\
\hline (D) Thrust $<0.977$ & 195 & 195 & 47.7 \\
\hline (E) Event Topology & 35 & 48 & 41.8 \\
\hline (F) Invariant Masses & 3 & 3.1 & 32.9 \\
\hline (G) $\frac{\Sigma|c \vec{p}|}{\sqrt{s}}<0.80$ & 0 & 1.6 & 32.5 \\
\hline
\end{tabular}


Table 2. Number of expected events for $e^{+} e^{-} \rightarrow \tilde{\gamma} \tilde{\gamma}$ according to the Babu-Mohapatra model, after subtracting the total error. The numbers in parentheses correspond to the search for $M_{\tilde{\gamma}}<10 \mathrm{GeV} / \mathrm{c}^{2}$ where Selection D was removed and Selection G was modified (see text).

\begin{tabular}{|c|c|c|c|}
\hline \multirow{2}{*}{$\begin{array}{l}\text { Masses } \\
\left(\mathrm{GeV} / \mathrm{c}^{2}\right)\end{array}$} & \multicolumn{3}{|c|}{ Expected Number of Events } \\
\hline & $M_{\tilde{e}}=40$ & $M_{\tilde{e}}=70$ & $M_{\tilde{e}}=100$ \\
\hline$M_{\tilde{\gamma}}=5$ & $0 \quad(13)$ & $0 \quad(5)$ & $0 \quad(2)$ \\
\hline$M_{\tilde{\gamma}}=8$ & $6 \quad(33)$ & $3 \quad(15)$ & $1(8)$ \\
\hline$M_{\tilde{\gamma}}=10$ & 26 & 11 & 5 \\
\hline$M_{\tilde{\gamma}}=20$ & 42 & 18 & 8 \\
\hline$M_{\tilde{\gamma}}=30$ & 19 & 8 & 3 \\
\hline$M_{\tilde{\gamma}}=40$ & 6 & 2 & 1 \\
\hline
\end{tabular}




\section{References}

[1] R. Davis et al. in Neutrino '88 Proc. of the 13th Int. Conf., Boston, Mass., 1988, edited by J. Schneps, T. Kafka, W. Mann and P. Nath (World Scientific, Singapore, 1989), p. 518.

[2] See for example the Review of Solar Neutrino Experiments by A. B. McDonald, in Vol. 1, The Vancouver Meeting, Particles and Fields 1991, edited by D. Axen, D. Bryman and M. Comyn. The recent results reported by the GALLEX experiment (P. Anselmann, et al, Phys. Lett. B285 (1992) 376) make the neutrino magnetic moment hypothesis less probable, but they are not accurate enough to rule it out. See for example K. S. Babu et al, Phys. Rev. D44, (1991) 2265.

[3] K. S. Babu and R. N. Mohapatra, Phys. Rev. D42 (1990) 3778; see also H. E. Haber and G. L. Kane, Physics Reports 117 (1985) 75.

[4] M. Davier, Proceedings of the Joint International Lepton-Photon Symposium \& Europhysics Conference on High Energy Physics(1991), Vol. II, p. 153, edited by S. Hegarty, K. Potter and E. Quercigh, and references therein.

[5] C. Hearty et al., Phys. Rev. D39, (1989) 3207 and references therein.

[6] OPAL Collaboration, K. Ahmet, et al., Nucl. Instr. and Methods A305 (1991) 275.

[7] J. Allison et al., Nucl. Instr. and Methods A317 (1992) 47;

J. Allision et al, Comp. Phys. Comm. 47 (1987) 55;

R. Brun et al, Geant3, Report DD/EE/84-1 CERN(1989).

[8] T. Sjöstrand, Computer Phys. Comm 39 (1986) 347;

T. Sjöstrand and M. Bengtsson, Computer Phys. Comm. 43 (1987) 367;

T. Sjöstrand, Int. J. of Mod. Phys. A3 (1988) 751.

[9] S. Jadach, B. F. L. Ward and Z. Wa̧s, Comp. Phys. Comm. 66 (1991) 276;

S. Jadach, B. F. L. Ward and Z. Wa̧s, Comp. Phys. Comm. 64 (1990) 275.

[10] M. Bohm, A. Denner and W. Hollik, Nucl. Phys. B304 (1988) 687;

F. A. Berends, R. Kleiss and W. Hollik, Nucl. Phys. B304 (1988) 712.

[11] F. A. Berends, P. H. Daverveldt and R. Kleiss, Nucl. Phys. B253 (1985) 421;

P. H. Daverveldt, Thesis, Rijksuniversiteit Leiden (unpublished).

[12] OPAL Collaboration, P. Acton et al., Phys. Lett. B287 (1992) 389.

[13] OPAL Collaboration, M. Z. Akrawy, et al., Phys. Lett. B263 (1991) 311.

[14] OPAL Collaboration, M.Z. Akrawy, et al., Phys. Lett. B253 (1991) 511. 


\section{Figure Captions}

Figure 1 (a) Feynman diagrams for photino production in $e^{+} e^{-}$annihilations by the Babu-Mohapatra mechanism.

(b) Feynman diagrams for the decays of the photinos via intermediate massive scalar particles $\tilde{e}, \tilde{\mu}$ or $\tilde{\tau}^{c}$, where $\tilde{\tau}^{c}$ is the supersymmetric scalar partner of the right handed $\tau^{+}$.

Figure 2 The thrust distribution for events remain after Selections A, B and C. The OPAL data are represented by the points with error bars. The shaded region shows the background, a mixture of multihadrons, lepton pairs and four-lepton final states, normalized to the number of multi-hadronic events in the data sample. The open histogram displays the unnormalized thrust distribution for a signal with $M_{\tilde{\gamma}}=20 \mathrm{GeV} / \mathrm{c}^{2}$ and $M_{\tilde{\epsilon}}=70 \mathrm{GeV} / \mathrm{c}^{2}$.

Figure 3 Distribution of $\Sigma|c \vec{p}| / \sqrt{s}$ after Selections A through F. The three remaining data events are indicated by stars. The signal expectation assuming $M_{\tilde{\gamma}}=$ $20 \mathrm{GeV} / \mathrm{c}^{2}$ and $M_{\tilde{e}}=70 \mathrm{GeV} / \mathrm{c}^{2}$ is represented by the open histogam and the background expectations by the shaded histograms.

Figure 4 95\% CL exclusion contour in the $M_{\tilde{\gamma}}$ vs $M_{\tilde{e}}$ plane deduced from this search. The region allowed by the Babu-Mohapatra model is indicated. 
(a)
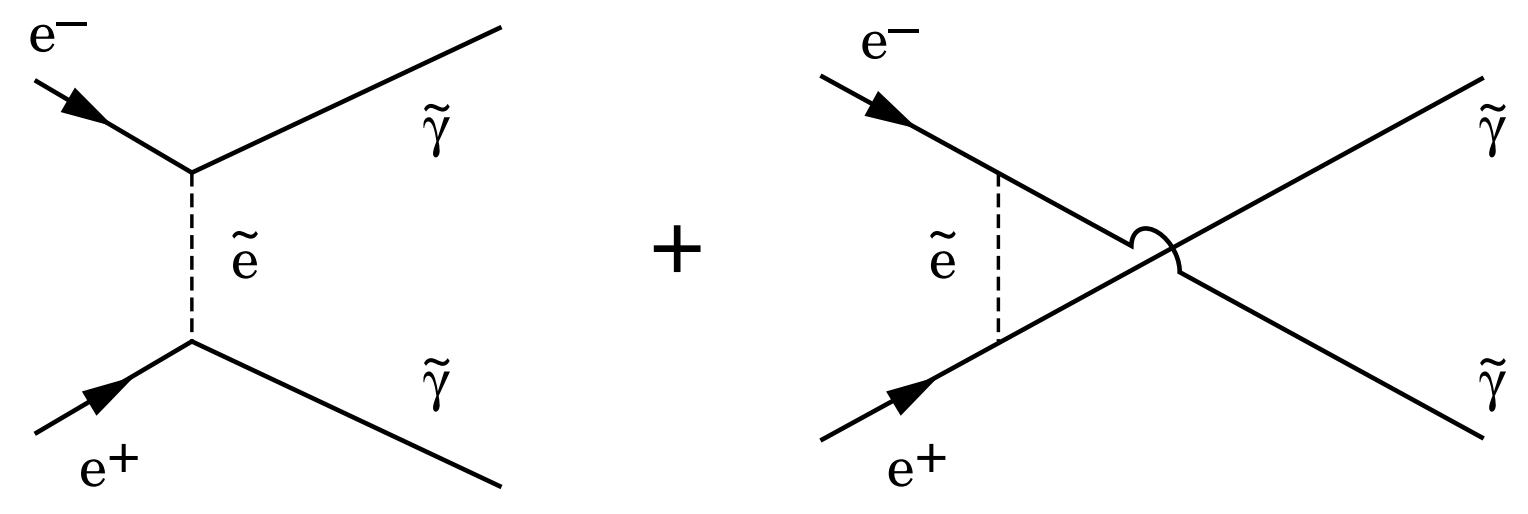

(b)
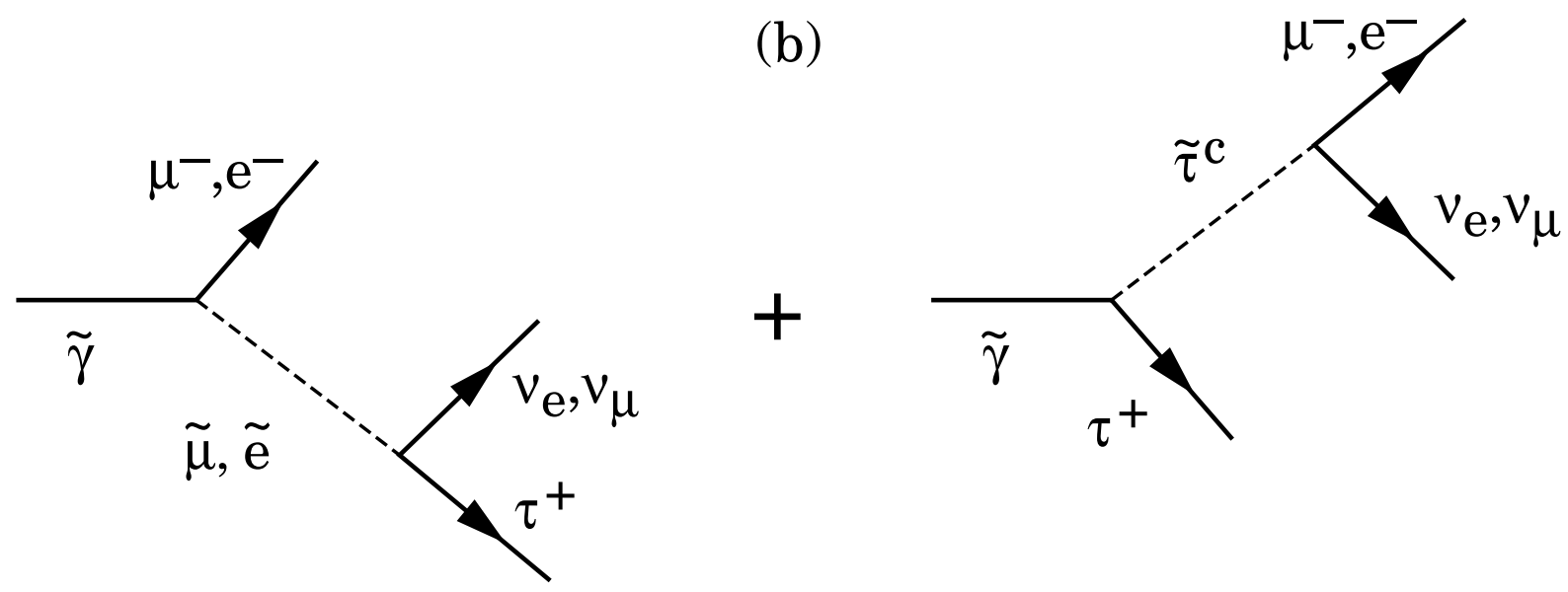

Fig. 1 


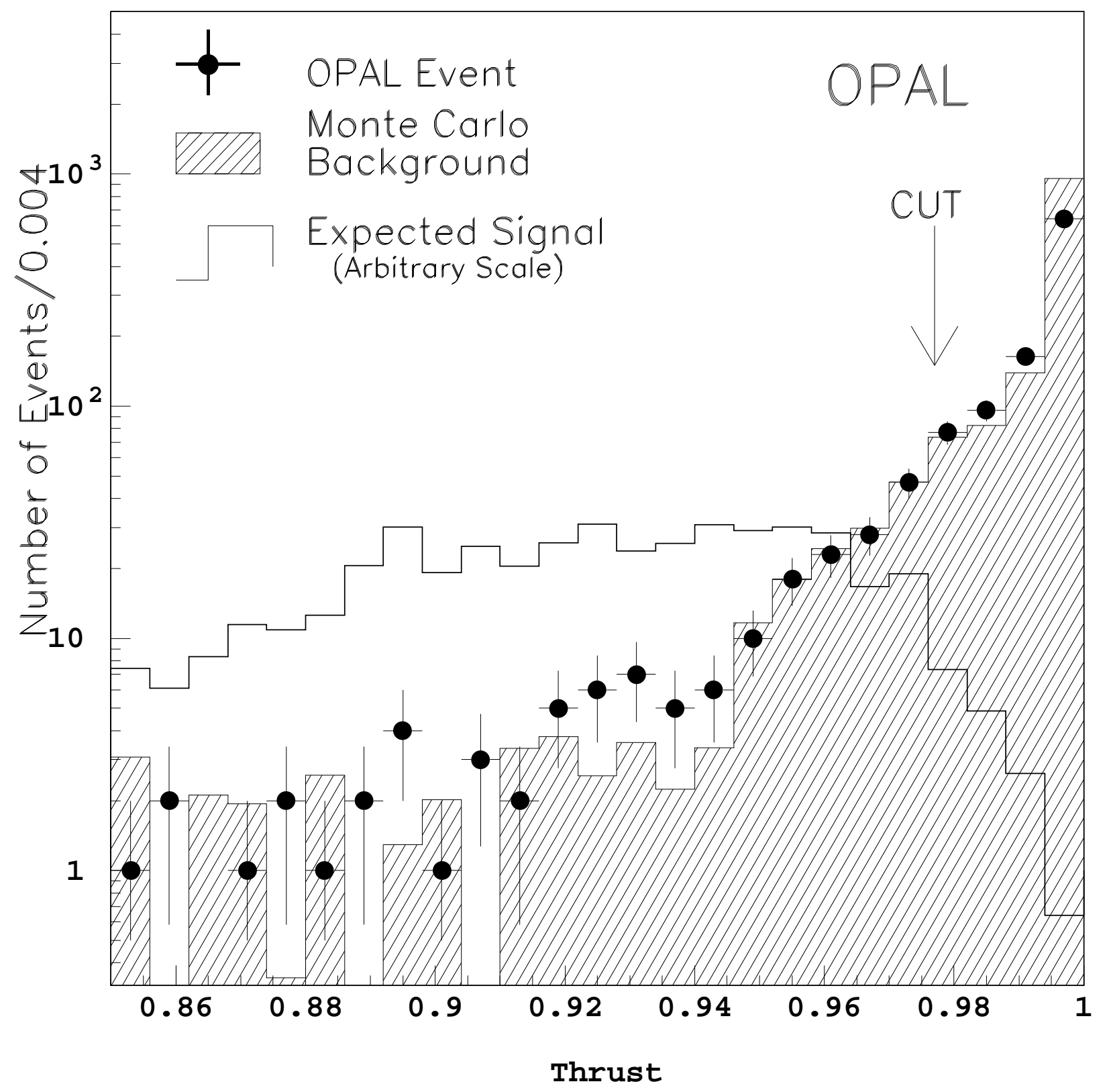

Fig. 2 


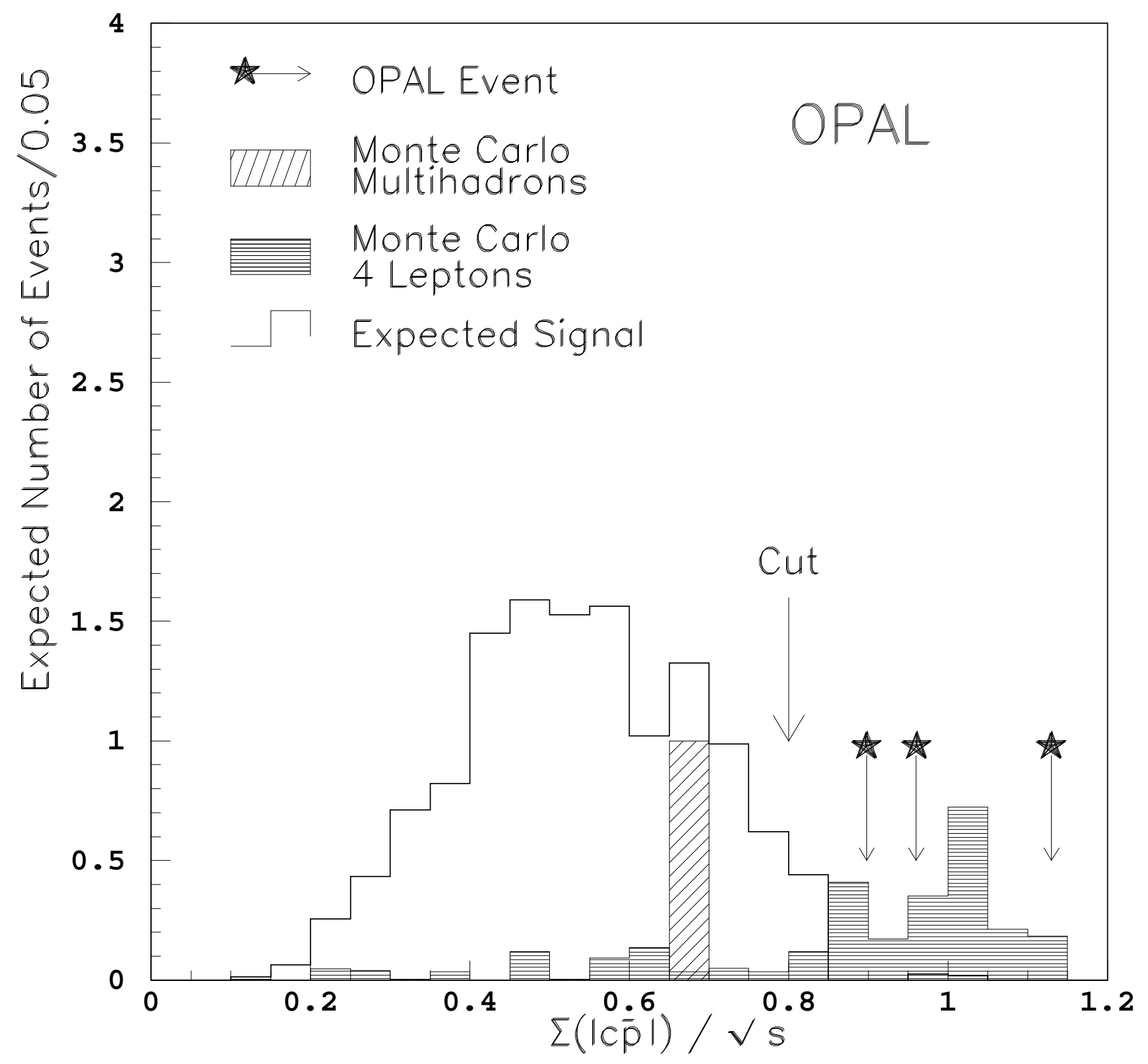

Fig. 3 


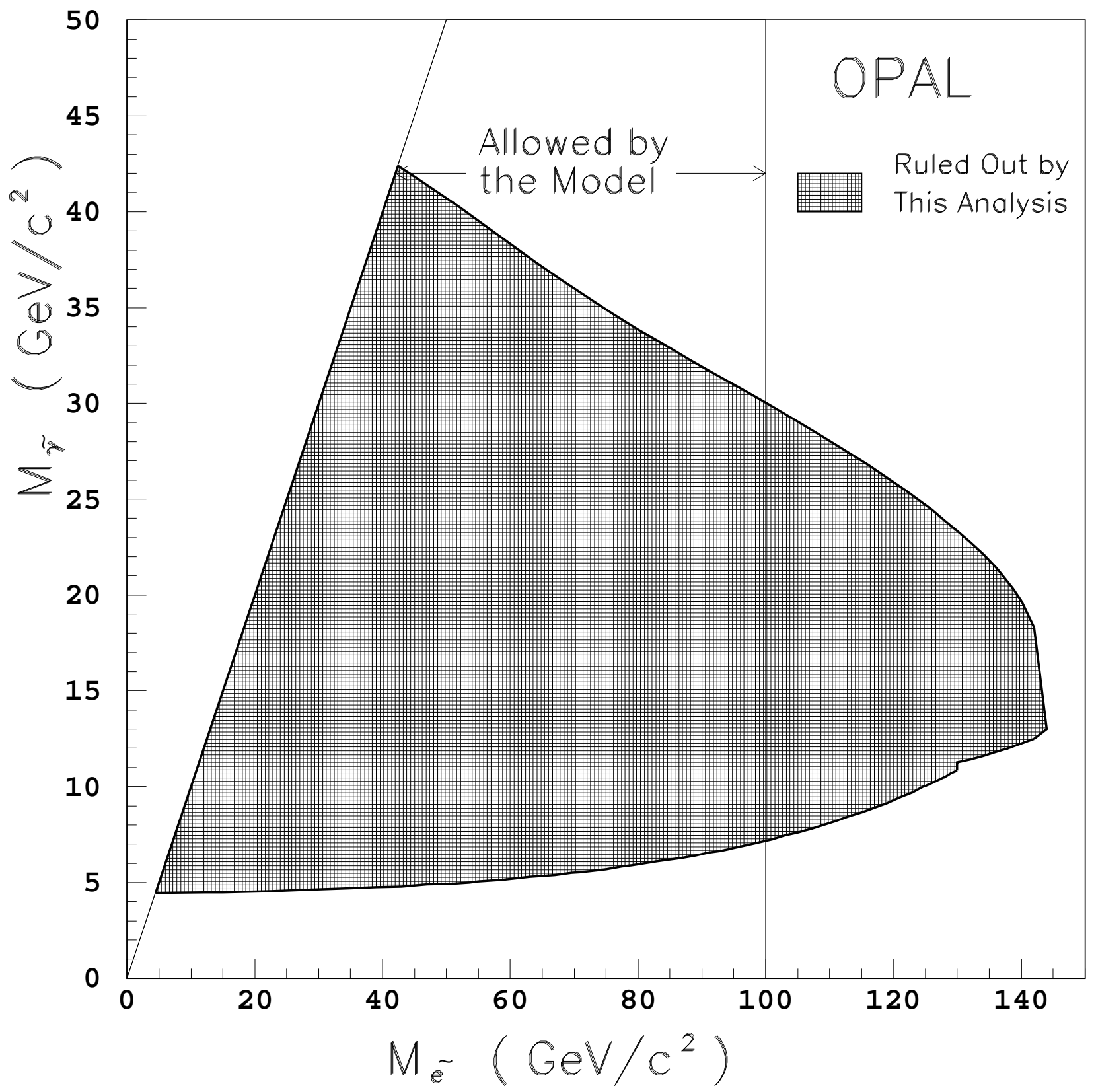

Fiq. 4 